\section{Corticostriatal and mesocortical dopamine systems: do species differences matter?}

\author{
Yoland Smith, Thomas Wichmann and Mahlon R. DeLong
}

Gordon M. G. Shepherd recently wrote a stimulating Review (Corticostriatal connectivity and its role in disease. Nature Rev. Neurosci. 14, 278-291 (2013) $)^{1}$ that provides novel perspectives on the potential role of the corticostriatal systems in neuropsychiatric disorders ranging from autism and schizophrenia to Parkinson's disease. However, we believe that important differences between the rodent and primate brain are not sufficiently discussed in this Review.

Dr Shepherd points out that the striatum is innervated by two different populations of cortical neurons - the intratelencephalic (IT) neurons and the pyramidal tract (PT) neurons - and discusses evidence for the hypothesis that disturbances in firing patterns of IT and PT neurons might contribute to human neuropsychiatric disorders. This discussion is based almost exclusively on data from the rodent literature and on the a Pyramidal tract

\section{Rodents}

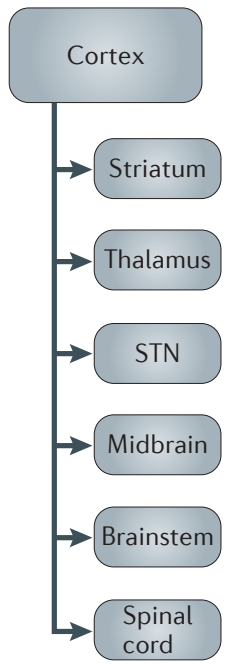

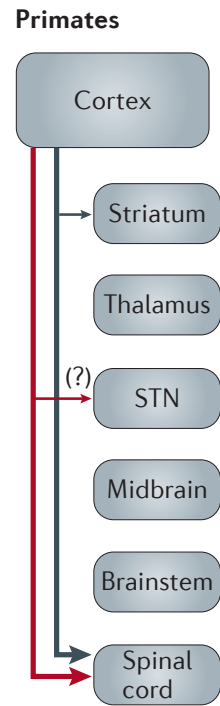

explicit assumption of an "evolutionary conservation of IT and PT organization across species and cortical areas". However, the literature suggests that there are, in fact, substantial species differences in the organization of the PT system. In rodents, the axons of cortical PT neurons branch heavily to innervate subcortical targets, such as the striatum, thalamus, subthalamic nucleus, ventral midbrain and brain stem $^{2}$. As shown in FIG. 1a, PT neurons are differently organized in primates: although the axons of a small subset of PT neurons sparsely branch to the striatum and claustrum, these neurons do not innervate other forebrain or midbrain regions ${ }^{3}$. Furthermore, careful studies in nonhuman primates have indicated that the electrophysiological and functional properties of cortical PT neurons in the primary motor cortex (M1) differ strikingly from those of corticostriatal neurons ${ }^{4-6}$.

\section{b Cortical dopamine}

Rodents

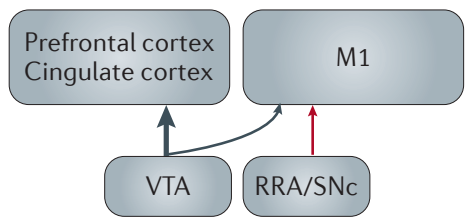

Primates

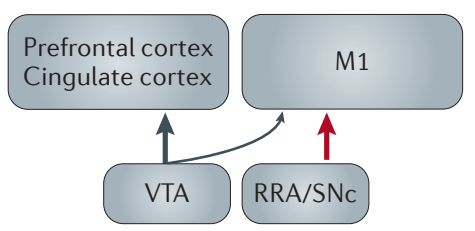

Importantly, recent data have shown that the electrophysiological activity of primate PT neurons is strongly altered in the (resting) parkinsonian state, whereas the electrophysiological activity of corticostriatal neurons is not affected ${ }^{6}$.

Species differences should also be considered when discussing dopaminergic projections to the cerebral cortex (FIG. $1 \mathrm{~b}$ ). In rodents, mesocortical dopamine projections arise almost exclusively from the ventral tegmental area (VTA) and terminate mostly in prefrontal regions. The primary motor cortex, where a large population of PT neurons is located, is only sparsely innervated by these fibres. By contrast, primates (including humans) have gained a substantial dopaminergic innervation of M1 and related motor cortices ${ }^{7,8}$; this innervation has arisen in large part from the substantia nigra pars compacta $(\mathrm{SNc})$ and the retrorubral area (RRA) ${ }^{9,10}$, thereby suggesting a prominent role of cortical dopamine on PT neurons in the M1 of primates, but not in rodents. These species differences in cortical dopamine projections might be important for our understanding of the pathophysiology of Parkinson's disease, in which neurons in the SNc and RRA are far more affected by the neurodegenerative process than those in VTA.

Thus, it seems that the organization of the corticostriatal system(s) and its modulatory dopaminergic inputs has undergone substantial evolutionary change. We believe that the resulting species differences deserve attention when interpreting data from animal studies because they affect our understanding of the neural substrates of normal behaviour and of the physiological disturbances that underlie neuropsychiatric disorders in humans.

Yoland Smith and Thomas Wichmann are at the Yerkes National Primate Research Center, the Morris UDALL Center of Excellence for Parkinson's Disease and the Department of Neurology, Emory University, 954 Gatewood Rd NE, Atlanta, Georgia 30322, USA.

Mahlon R. DeLong is at the Morris UDALL Center of Excellence for Parkinson's Disease and the Department of Neurology, Emory University, Woodruff Memorial Building, Pierce Drive, Atlanta, Georgia 30322, USA.

Corresponding author Y.S. e-mail: ysmit01@emory.edu

doi: 10.1038/nrn3469-c1 Published online 4 December2013

Figure 1 | Highly simplified diagram of key anatomical differences between rodents and primates with regard to pyramidal tract neurons (a) and the sources of dopamine innervation of prefrontal/cingulate and M1 cortices (b). Red and black arrows are used to show projections from distinct neuronal populations. The thickness of arrows in these diagrams indicates relative differences in the extent of target innervation by the afferent inputs. For simplicity, some corticostriatal neuron subtypes are not shown. M1, primary motor cortex; RRA, retrorubral area; SNc, substantia nigra pars compacta; STN, subthalamic nucleus; VTA, ventral tegmental area.
1. Shepherd, G. M. Corticostriatal connectivity and its role in disease. Nature Rev. Neurosci. 14, 278-291 (2013).

2. Kita, T. \& Kita, H. The subthalamic nucleus is one of multiple innervation sites for long-range corticofugal axons: a single-axon tracing study in the rat. J. Neurosci. 32, 5990-5999 (2012). 
3. Parent, M. \& Parent, A. Single-axon tracing study of corticostriatal projections arising from primary moto cortex in primates. J. Comp. Neurol. 496, 202-213 (2006).

4. Bauswein, E., Fromm, C. \& Preuss, A. Corticostriatal cells in comparison with pyramidal tract neurons: contrasting properties in the behaving monkey. Brain Res. 493, 198-203 (1989).

5. Turner, R. S. \& DeLong, M. R. Corticostriatal activity in primary motor cortex of the macaque. J. Neurosci. 20, 7096-7108 (2000).

6. Pasquereau, B. \& Turner, R. S. Primary motor cortex of the parkinsonian monkey: differential effects on the spontaneous activity of pyramidal tract-type neurons. Cereb. Cortex 21, 1362-1378 (2011)

7. Berger, B., Gaspar, P. \& Verney, C. Dopaminergic innervation of the cerebral cortex: unexpected differences between rodents and primates. Trends Neurosci. 14 21-27 (1991)

8. Luft, A. R. \& Schwarz, S. Dopaminergic signals in primary motor cortex. Int. J. Dev. Neurosci. 27, 415-421 (2009).

9 Lewis, D. A Campbell, M. J., Foote, S. L. Goldstein, M. \& Morrison, J. H. The distribution of tyrosine hydroxylase-immunoreactive fibers in primate neocortex is widespread but regionally specific.

J. Neurosci. 7, 279-290 (1987).
10. Williams, S. M \& Goldman-Rakic, P. S. Widespread origin of the primate mesofrontal dopamine system. Cereb. Cortex 8, 321-345 (1998).

Acknowledgements

The authors are supported by US National Institute of Health grants P50NS071669 (T.W., M.R.D. and Y.S.) and R01NS037948, NS083386, R01NS071074 and RR00165 (T.W. and Y.S.).

\section{Competing interests statement}

The authors declare no competing interests. 